\title{
Anomalous quantum Hall conductivity and resonances in coupled layers.
}

\author{
A. L. Chudnosvkiy and S. E. Ulloa \\ Department of Physics and Astronomy and Condensed Matter and Surface Sciences Program \\ Ohio University, Athens, Ohio 45701-2979
}

\begin{abstract}
A two-layer system coupled via tunneling and with different carrier masses in each layer is investigated in the integer quantum Hall regime. Striking deviations of the one-layer Hall conductivity from the usual quantization are found, if resonance between Landau levels of different layers is achieved. The appearance of negative jumps in the Hall conductivity is also predicted under suitable conditions. The results obtained are robust against disorder in the system.
\end{abstract}

Recent progress in nanotechnology has made possible the creation of electronic systems with unusual and interesting properties. Prominent examples include spatially separated electronic layers coupled by tunneling [1] or only via Coulomb interactions [2], quantum dot arrays in different configurations [3, [4], or even combinations of layers and quantum dots [5,6]. These artificial systems provide interesting analogues to natural systems, although with different characteristic length and energy scales. On the other hand, the artificially constructed systems may serve as a laboratory to discover and utilize in practice new physical effects, hardly seen in naturally occurring solids.

The aim of this paper is to draw attention to qualitatively new physical effects which are predicted to occur in the integer quantum Hall $(\mathrm{QH})$ regime for a system of two conducting layers coupled via tunneling and with charge carriers of different effective masses. In the inset to Fig. 1. we suggest a suitable experimental configuration for such a system. The spatial separation of the layers allows for experimental measurements of the quantum Hall resistance of each layer independently, which would exhibit the well-known plateau structure versus the magnetic field (or the Fermi energy). The presence of the other layer coupled via tunneling, however, leads to quantum interference effects that result in the deviation of the resistivity plateaus from quantized values, if two Landau levels of the different layers happen to be close in energy (in resonance).

By applying a bias voltage $V_{0}$, as shown in Fig. 1, one can "slide" the system of LL's of one layer with respect to that of the other, thus changing the value of the one-layer resistivity on the plateau, and producing a tunable quantum Hall resistor. One should mention that such 'gated' configurations have been successfully implemented in a number of clever magnetocapacitance and transport experiments [1, 6, 8], and are in great part the motivation for this work. Different realizations can also be proposed and are experimentally feasible, including one with dopants of different kind (donors and acceptors) on each layer, as electrons and holes would have substantially different masses (similar systems have been recently studied [7]), or one with different semiconductor materials across the interface, such as InAs-GaAs, to give different-mass carriers in the conduction bands. One of the particularly interesting realizations of such two-layer system is a two-dimensional electron system (2DES), coupled via a tunneling barrier to a planar array of quantum dots (QDs) [6]. In the limit of close in-plane separation between dots, to allow direct electron tunneling, the 2DES-QDs structure would effectively reduce to that of two layers with different effective masses.

In a two-layer system, the longitudinal and transverse resistivities become matrices with components reflecting the position of the measuring contacts, for example, $\rho_{x y}^{\alpha \beta, \gamma \delta}$, where $\alpha, \beta, \gamma, \delta$ can be either $l$ or $h$, denoting the 'light-' or 'heavy-mass' particle layer, as shown in the inset to Fig. 2(a). It is important, that each component of the resistivity tensor can be measured independently by switching the measuring contacts. In what follows, we shall discuss the properties of the conductivity matrix $\sigma_{x y}^{\alpha \beta, \gamma \delta}=\left(\rho_{x y}^{-1}\right)^{\alpha \beta, \gamma \delta}$, which can be calculated directly from the experimental data. Similarly, one can obtain any desired $\rho_{x y}$ component from the theoretical calculations of $\sigma_{x y}$, as we illustrate below.

Under the resonant conditions above, the components of the transverse conductivity matrix exhibit striking behavior, as the Fermi level of the system moves through the level structure. First, the jumps deviate from the usual value, producing Hall conductivity plateaus at non-quantized values. Moreover, the jumps can be both positive and negative - that is, the value of the transverse conductivity measured in a given layer can change non-monotonically (see Fig. 2(b)). Finally, if the layers have different effective mobilities (with corresponding different widths of their LL's), singularities in the density of states may occur, leading in turn to additional features in the components of the Hall conductivity in each layer. The prediction of non-quantized and even negative jumps of the transverse conductivity in the two-layer QH system under resonant conditions is one of the main results of this paper.

One can understand the deviation from exact quantization in each layer as resulting from the shift of LL energies due to the coupling between the layers. In a clean system, the position of the states is shifted due to the interlayer 
tunneling $t$, as defined by the well-known expression,

$$
\epsilon_{k}^{ \pm}=\frac{1}{2}\left(E_{k}^{l}+E_{k}^{h} \pm\left[\left(E_{k}^{l}-E_{k}^{h}\right)^{2}+4 t^{2}\right]^{1 / 2}\right)
$$

where $E_{k}^{l}=(k+1 / 2) \Omega$ is the energy of the unperturbed (no tunneling) LL in the layer of light particles, while $\Omega$ denotes the cyclotron frequency in this "l-layer", $E_{k}^{h}=E_{0}+(k+1 / 2) \Omega / M$ is the energy of the unperturbed LL in the heavy particle layer ( $M$ is the mass ratio between heavy and light particles, and $E_{0}$ is the energy offset between the two systems of LL's due to the voltage applied). Here and in what follows we use units with $h=1$ and $m_{e}=1$. Using the Kubo formula, up to corrections of order $t / \Omega$, one obtains the following expression for the jump in transverse conductivity around the $\mathrm{n}$-th Landau level in the l-layer

$$
\Delta_{n} \sigma_{x y}^{l l, l l}=\lim _{\omega \rightarrow 0} \frac{\Omega^{2}}{2 \omega}\left[\frac{1}{\epsilon_{n+1}^{l}-\epsilon_{n}^{l}-\omega}-\frac{1}{\epsilon_{n+1}^{l}-\epsilon_{n}^{l}+\omega}\right] .
$$

In the usual case, this expression yields one conductivity quantum, if the degeneracy of the Landau level (which equals $\Omega$ in these units) is equal to the separation between neighbor LL's, $\epsilon_{n+1}^{l}-\epsilon_{n}^{l}$, as is the case in the absence of tunneling. The tunneling between the layers, however, leads to $\epsilon_{n+1}^{l}-\epsilon_{n}^{l} \neq \Omega$, and hence to the deviation of the jump from one conductivity quantum of order $t / \Omega$. Another effect of tunneling is perhaps more subtle, as the conductivity involves in general not only LL's of the same layer, but also those of different layers. This is reflected in the presence of energy differences of the type $\epsilon_{n+1}^{l}-\epsilon_{n}^{h}$ in the general expression for the Hall conductivity (not shown). Sliding one system of LL's with respect to the other, one can achieve the resonance between the energies $\epsilon_{n+1}^{l}$ and $\epsilon_{n}^{h}$. Close to that resonance, additional nonquantized jumps in the transverse conductivity of each layer appear, the sign of the jumps being defined by the sign of the difference $\epsilon_{n+1}^{l}-\epsilon_{n}^{h}$. The jumps can, therefore, be negative, as well as positive.

One should emphasize, however, that the topologically-expected quantization condition is fulfilled for the combination

$$
\sigma_{x y}^{l l, l l}+\sigma_{x y}^{l l, h h}+\sigma_{x y}^{h h, h h}+\sigma_{x y}^{h h, l l} .
$$

This quantization conditions follows naturally, if one considers a two-layer system, connected fully in parallel. The resulting conductivity of the effective one layer system should be quantized in the traditional way, and it remains unaffected by the singularities due to disorder.

At large tunneling $(t \sim \Omega)$, the components $\sigma_{x y}^{l l, l l}$ and $\sigma_{x y}^{h h, h h}$ cease to reflect the measured conductances in each layer because of the strong interlayer coupling. In the limit of very large tunneling, the measurements would give only the effective layer results, given by the trace of the Hall conductivity tensor and always well-quantized.

To obtain a full analytical description of the system considered, we generalize the replica nonlinear sigma model approach to the integer QH effect developed by Pruisken [11] to the case of a two-layer system with different effective masses. The Hamiltonian of the two-layer model we are interested in can be written as

$$
\begin{aligned}
H= & \sum_{r}\left\{\hat{a}_{r}^{+}\left(\hat{\pi}_{\mu} \hat{\pi}^{\mu}+V_{l}\right) \hat{a}_{r}+\hat{c}_{r}^{+}\left[E_{0}+\frac{1}{M} \hat{\pi}_{\mu} \hat{\pi}^{\mu}+V_{h}\right] \hat{c}_{r}\right. \\
& \left.+t\left(\hat{a}_{r}^{+} \hat{c}_{r}+\hat{c}_{r}^{+} \hat{a}_{r}\right)\right\},
\end{aligned}
$$

where the fermionic operators $\hat{a}$ and $\hat{c}$ refer to the states in the two different layers, the operator $\hat{\pi}_{\mu} \hat{\pi}^{\mu}$ describes the kinetic energy in a magnetic field $\left(\hat{\pi}^{\mu} \equiv-i \nabla_{\mu}-A_{\mu}, \mu=x, y, \hat{\pi}_{\mu}=\left(\hat{\pi}^{\mu}\right)^{+}\right), t$ is the tunneling matrix element between the two layers, and $E_{0}$ describes the relative position of the bottom of the band of layer $c$ with respect to the layer $a$. The mass of the electrons in layer $a$ is taken as unity, so that $M \geq 1$ is the relative mass of the 'heavier' quasiparticles in layer $c$. The random gaussian-distributed potentials $V_{l}(r)$ and $V_{h}(r)$ describe the disordered scattering in the $l$-, and the $h$-layer, respectively. The only nonvanishing correlators of the random potentials are $\left\langle V_{\alpha}(r) V_{\alpha}\left(r^{\prime}\right)\right\rangle=\frac{1}{2 \pi \nu \tau_{\alpha}} \delta\left(r-r^{\prime}\right)$, where $\nu$ is the density of states of a LL, $\alpha=l$, $h$, and $\tau_{l}$ and $\tau_{h}$ are the mean free times for a particle in the light- and heavy-mass layers, respectively $(1 / \tau$ is the LL width). Note, that we consider here a model without possible disorder in the tunneling between the layers, $t$. The model with disorder in the tunneling belongs to a different class and will be considered in the future.

Following the ideas of Ref. [11], we introduce in the two-layer system the basis of grassmanian vectors $\Phi=\left(\Psi_{l}, \Psi_{h}\right)^{T}$, where the subscripts $l$ and $h$ relate to the layers. Each of the grassmanian components consists in turn of two variables, relating to the advanced and retarded sectors, $\Psi_{l, h}=\left(\psi_{l, h}^{A}, \psi_{l, h}^{R}\right)$. 
Employing the replica trick to average over the disorder, we decouple the resulting four-fermionic terms with the help of the Hubbard-Stratonovich transformation, and integrate out the fermionic degrees of freedom. The decoupling matrix field has the structure

$$
\hat{\mathbf{Q}}=\left(\begin{array}{cc}
\hat{Q} & 0 \\
0 & \hat{R}
\end{array}\right)
$$

where the $2 n \times 2 n$ hermitian matrices $\hat{Q}$ and $\hat{R}$ facilitate the decoupling of the four-fermionic terms in the 'light' and 'heavy' sectors, respectively. According to the standard procedure [11], we look for a mean-field solution for the matrix fields $\hat{Q}$ and $\hat{R}$ of the form $\hat{Q}=q \sigma^{\mathbf{z}} \otimes \mathbf{1}_{\mathbf{n}}, \hat{R}=r \sigma^{\mathbf{z}} \otimes \mathbf{1}_{\mathbf{n}}$, where $\sigma^{\mathbf{z}}$ is a Pauli matrix. The mean-field equations for $q$ and $r$ are then obtained in the form

$$
\begin{aligned}
& q=\sum_{k} \frac{1}{D_{k}}\left\{\frac{r}{2 \tau_{h}}\left[\frac{q r}{4 \tau_{l} \tau_{h}}-\left(E_{k}^{l}-\epsilon_{F}\right)\left(E_{k}^{h}-\epsilon_{F}\right)+t^{2}\right]\right. \\
& \left.+\left(E_{k}^{h}-\epsilon_{F}\right)\left[\frac{q}{2 \tau_{l}}\left(E_{k}^{h}-\epsilon_{F}\right)+\frac{r}{2 \tau_{h}}\left(E_{k}^{l}-\epsilon_{F}\right)\right]\right\}
\end{aligned}
$$

where

$$
\begin{aligned}
& D_{k}=\left[\frac{q r}{4 \tau_{l} \tau_{h}}-\left(E_{k}^{l}-\epsilon_{F}\right)\left(E_{k}^{h}-\epsilon_{F}\right)+t^{2}\right]^{2} \\
& +\left[\frac{q}{2 \tau_{l}}\left(E_{k}^{h}-\epsilon_{F}\right)+\frac{r}{2 \tau_{h}}\left(E_{k}^{l}-\epsilon_{F}\right)\right]^{2},
\end{aligned}
$$

and with a similar equation for $r$. In the sum over the $k$-Landau levels, only the contribution of the level closest to the Fermi energy, $\epsilon_{F}$, should be kept.

Solving Eq. (6) and the analogous one for $r$ numerically, we can calculate the transverse conductivity tensor $\hat{\sigma}_{x y}$. Here, we concentrate on the behavior of the diagonal components of the transverse conductivity tensor, which correspond to the measurements of the conductivity when transverse contacts are applied in each layer, while the current is injected into both layers (see inset in Fig. 2(a)). If there is no resonance between LL's of different layers, the behavior of the transverse conductivity in each layer is quite normal, as the conductivity jumps by one quantum $\left(e^{2} / h\right)$ each time the Fermi energy passes a Landau level of the given layer.

On the other hand, if there is a resonance between the LL's of different layers, the behavior of the transverse conductivity changes drastically, exhibiting different regimes as function of the disorder strength in different layers. There are two types of resonances with different behavior of $\hat{\sigma}_{x y}$ : (i) resonance between LL's with the same quantum number, $E_{n}^{l}=E_{n}^{h}$; and (ii) resonance between LL's with quantum numbers differing by $1, E_{n}^{l}=E_{n \pm 1}^{h}$.

Both types of resonance are affected by the disorder, and in a singular way for strong disorder. Weak disorder will produce level broadening and a slight shift of the eigenenergies of the clean system, given by Eq. (1), but their splitting is still proportional to $t$ on resonance, and the corresponding states are mixtures of the light- and heavy-particle layer eigenfunctions.

In case (i) and weak disorder, as shown in Fig. 2(a), the transverse conductivity components, $\sigma_{x y}^{l l, l l}, \sigma_{x y}^{h h, l l}=\sigma_{x y}^{l l, h h}$ and $\sigma_{x y}^{h h, h h}$, experience a jump each time the Fermi level crosses one of the two eigenenergies of the spectrum, $\epsilon_{n}^{l}$ and $\epsilon_{n}^{h}$. Notice that due to the tunneling coupling between the layers, these eigenvalues are shifted with respect to the resonant energy $E_{n}^{l}=E_{n}^{h}$ by an amount proportional to $t$. The jumps of the transverse conductivity measured in each layer are non-quantized, with plateaus in each layer not equal to an integer number of quanta. Notice, however, that the sum of the values of the Hall conductivity in the two layers, corresponding to combination (3) is indeed wellquantized, as one would expect of the entire system based on general topological considerations [9, 10]. The departure from quantization of the conductivity plateaus in different layers grows with the difference in effective mass of the particles, with the higher conductivity plateau for the lighter particles. The width of the non-quantized plateau in Fig. 2(a) grows with the tunneling amplitude $t$ causing the splitting of the resonant LL's. The nonquantized plateau of the components of the conductivity imply a similarly nonquantized plateaus of the components of the resistivity, which would be explicitly observable in experiment (as an example, the dependence of $\rho_{x y}^{l l, l l}$ is shown in Figure 2(a)).

In the case (ii) of a resonance with different Landau index, the deviation of the transverse conductivity from the usual behavior is even more profound. Figure 2(b) shows the dependence of the transverse conductivity in each layer vs. Fermi energy position, in the case of the resonance $E_{n}^{l}=E_{n+1}^{h}+\delta$. A small deviation from the exact resonance is needed in order to see the middle plateau in Fig. 2(b) between the two nonquantized jumps, otherwise (for $\delta=0$ ) 
one would see only one jump (also nonquantized), which is the sum of the jumps around the levels $E_{n+1}^{h}$ and $E_{n}^{l}$. Here, similar to the previous case, the transverse conductivity in each layer jumps by non-quantized values when the Fermi energy crosses one of the shifted energy levels, $\epsilon_{n}^{l}$ and $\epsilon_{n+1}^{h}$. The jump of each component can, however, exceed one quantum, in which case the jump in the other layer will be negative, as to ensure the proper quantization of the conductivity trace. The amplitude of the jump of each component grows with the tunneling strength $t$, as shown in the inset. The tunneling determines also the width of the resonant region. For very different effective masses and large enough tunneling, such as $M=10, t=5$, two levels can be in the resonant region, such as $E_{n+1}^{h}$ and $E_{n}^{h}$, and two anomalous jumps in each layer can be observed. Naturally, the anomalous part of a jump (different from 1 or 0 conductivity quantum) decreases away from the resonant conditions. Experimentally, the tuning of the resonance can be achieved in principle by changing the bias voltage $V_{0}$ (or energy offset $E_{0}$ in our description). The sign of the jump of each component at a particular resonance is defined by the relative position of the resonant LL's. For example, in the case $E_{n}^{l} \approx E_{n+1}^{h}$, the jump of the component $\sigma_{x y}^{l l, l l}$ is negative at $E_{n+1}^{h}<E_{n}^{l}$, and it is positive for the opposite relation. Weak disorder in each layer softens the shape of the jumps.

For strong disorder, the situation changes even more, as it influences the position of the energy levels and the density of states. As mentioned above, the disorder results in broadening, given mathematically by the appearance of an imaginary part for each energy, e.g., $E_{k}^{l} \rightarrow E_{k}^{l}-i \frac{q}{2 \tau_{l}}$, which results in a shift and the appearance of imaginary parts in the energies $\epsilon^{ \pm}$in Eq. (11). For strong disorder, the energies $\epsilon^{ \pm}$exhibit square root singularities as functions of the Fermi energy. These in turn result in singular behavior of the density of states, and in the appearance of additional features in $\sigma_{x y}^{l l, l l}, \sigma_{x y}^{h h, h h}$, and other components in the case (i) (not shown). As for other jumps, the combination in (3) remains continuous and with well-quantized values, while the individual components have unusual features. For the resonance between the LL's with different quantum numbers, case (ii), the strong disorder produces just increasing broadening of each level.

We should notice that the solutions of the nonlinear equations for $q$ and $r$ (see Eq. (6)) are related to the structure of the density of states of the coupled system. The values of $q$ and $r$ change with the position of the Fermi energy, as shown in Fig. 3, for a typical case. Note that the gaps in $q\left(\epsilon_{F}\right)$ and $r\left(\epsilon_{F}\right)$ have the same origin as the gap in the density of states found for a 2DEG-QD system in Ref. [12]. Our results here should then provide a description of the transport properties for such a system [6].

It is important to notice that all of these results for $\hat{\sigma}_{x y}$, which have been obtained by treating the disorder on a mean-field level, are not affected by the fluctuations. To study this, we have also derived the nonlinear $\sigma$-model for the two-layer QH system. Details will be presented elsewhere 13. The fluctuation expansion around the mean-field solution leads to a nonlinear $\sigma$-model of the same class that the model for the single-layer system [11]. Here, the role of the coupling constants is played by the traces of longitudinal and transverse conductivity tensors. Analysis of the Ward identities shows that the diagonal components of the transverse conductivity tensor are coupled to a topologically invariant term only [11], and are therefore not affected by the fluctuations. As such, one finds the identity of the loop expansions for each component of the tensor $\hat{\sigma}_{x y}$, and for the transverse conductivity of the one layer system, up to a general prefactor. This in turn leads one to the conclusion that the results for each component of the transverse conductivity tensor, obtained at the mean-field level, are robust against the fluctuations.

We thank P. Pereyra for fruitful discussions. This work was supported in part by US Department of Energy Grant No. DE-FG02-91ER45334.

[1] J. P. Eisenstein, L. N. Pfeiffer, and K. W. West, Phys. Rev. Lett. 69, 3804 (1992)

[2] T. J. Gramila, J. P. Eisenstein, A. H. MacDonald, L. N. Pfeiffer, and K. W. West, Phys. Rev. Lett. 66, 1216 (1991)

[3] A. P. Alivisatos, Science 271, 933 (1996)

[4] R. Leon, P.M. Petroff, D. Leonard, and S. Fafard, Science 267, 1966 (1995)

[5] A. S. G. Thornton, T. Ihn, P. C. Main, L. Eaves, K. A. Benedict, M. Henini, Physica B, 249-251, 689 (1998)

[6] E. Ribeiro, E. Müller, T. Heinzel, H. Auderset, K. Ensslin, G. Medeiros-Ribeiro and P. M. Petroff, Phys. Rev. B 58, 1506 (1998)

[7] Y. Yaish, O. Prus, E. Buchstab, G. Ben Yoseph, and U. Sivan, Proceedings of the 24th International Conference on the Physics of Semiconductors, Jerusalem, 1998, to be published.

[8] B.T. Miller, W. Hansen, S. Manus, R.J. Luyken, A. Lorke, and J.P. Kotthaus, Phys. Rev. B 56, 6764 (1997) and references therein.

[9] See for example, The Quantum Hall Effect, R. E. Prange and S. M. Girvin, eds. (Springer, New York, 1987). 
[10] M. Kohmoto, Ann. Phys. (New York) 160, 343 (1985)

[11] A.M.M. Pruisken, Nucl. Phys. B235, 277 (1984)

[12] T. V. Shahbazyan and S. E. Ulloa, Phys. Rev. Lett. 79, 3478 (1997) and Phys. Rev. B 57, 6642 (1998)

[13] A. L. Chudnovskiy and S. E. Ulloa, unpublished.

FIG. 1. Experimental scheme for the measurements of the quantum Hall conductivity resonances. The voltage $V_{0}$ shifts LL ladders in the $l$ - and $h$-layers relative to one another.

FIG. 2. The transverse conductivity components vs Fermi energy at weak disorder, $\tau_{l}=1, \tau_{h}=10, M=5, t=3, \Omega=100$. The total conductivity corresponds to the expression (3). (a) The case of the resonance $E_{2}^{h}=E_{2}^{l}=5 \Omega / 2$. The dashed-dotted line shows the component $\rho_{x y}^{l l, l l}$ of the resistivity matrix, which illustrates the nonquantized plateaus of the resistivity. (b) The case of the resonance $E_{3}^{h}+\delta=E_{2}^{l}$. Arrows show the correspondence between the positions of the jumps and the energies of unshifted LL's. In the inset, a large tunneling case, $t=6$, is shown. The resonant region is larger and involves also the level $E_{2}^{h}$

FIG. 3. Typical solutions to the mean field equations, $q\left(\epsilon_{F}\right)$ (solid line) and $r\left(\epsilon_{F}\right)$ (dashed). Here, $\tau_{l}=0.01, \tau_{h}=1, M=5$, and $t=3$. The inset shows a case of smaller tunneling, $t=1$. 

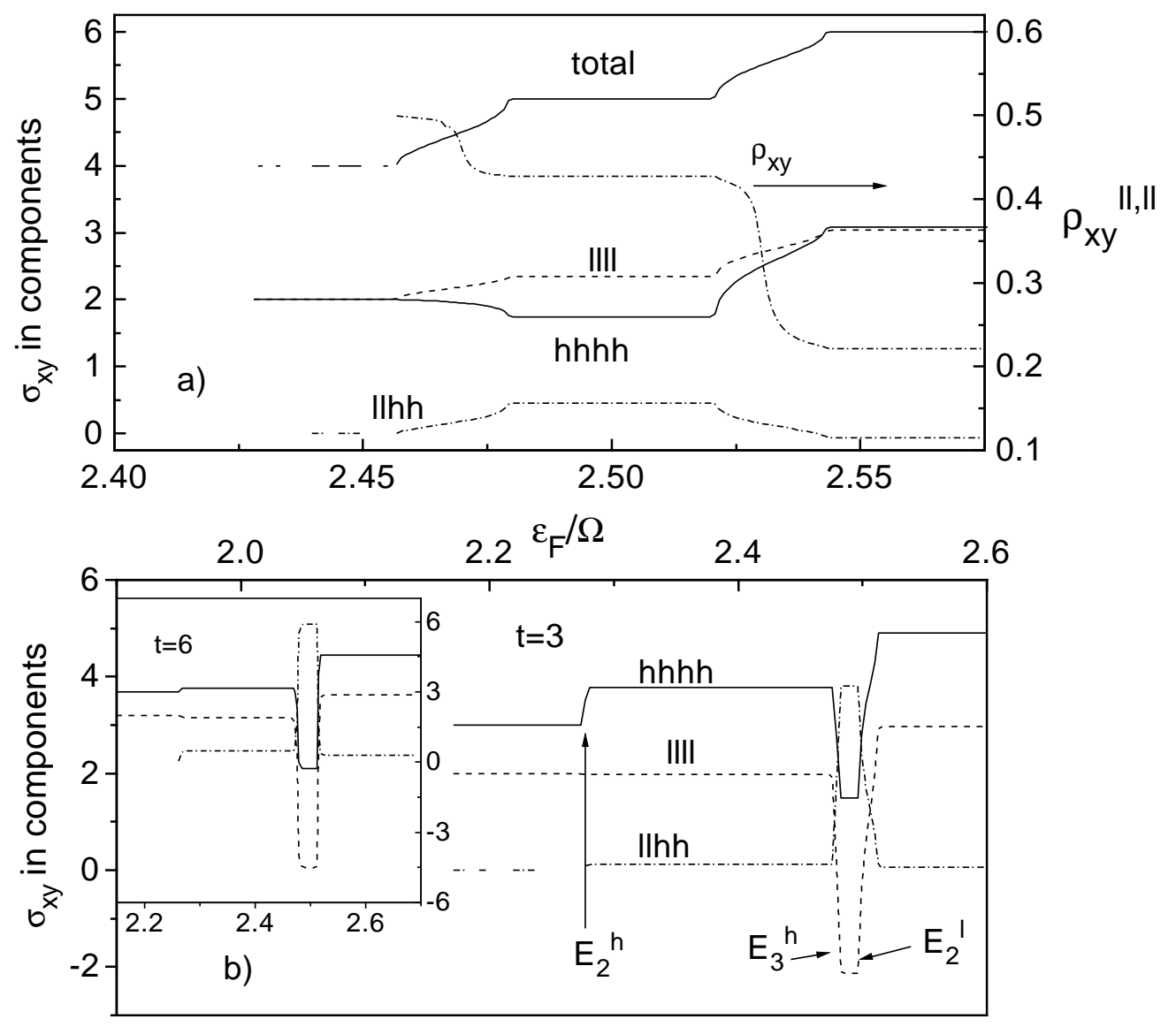

Figure 2 


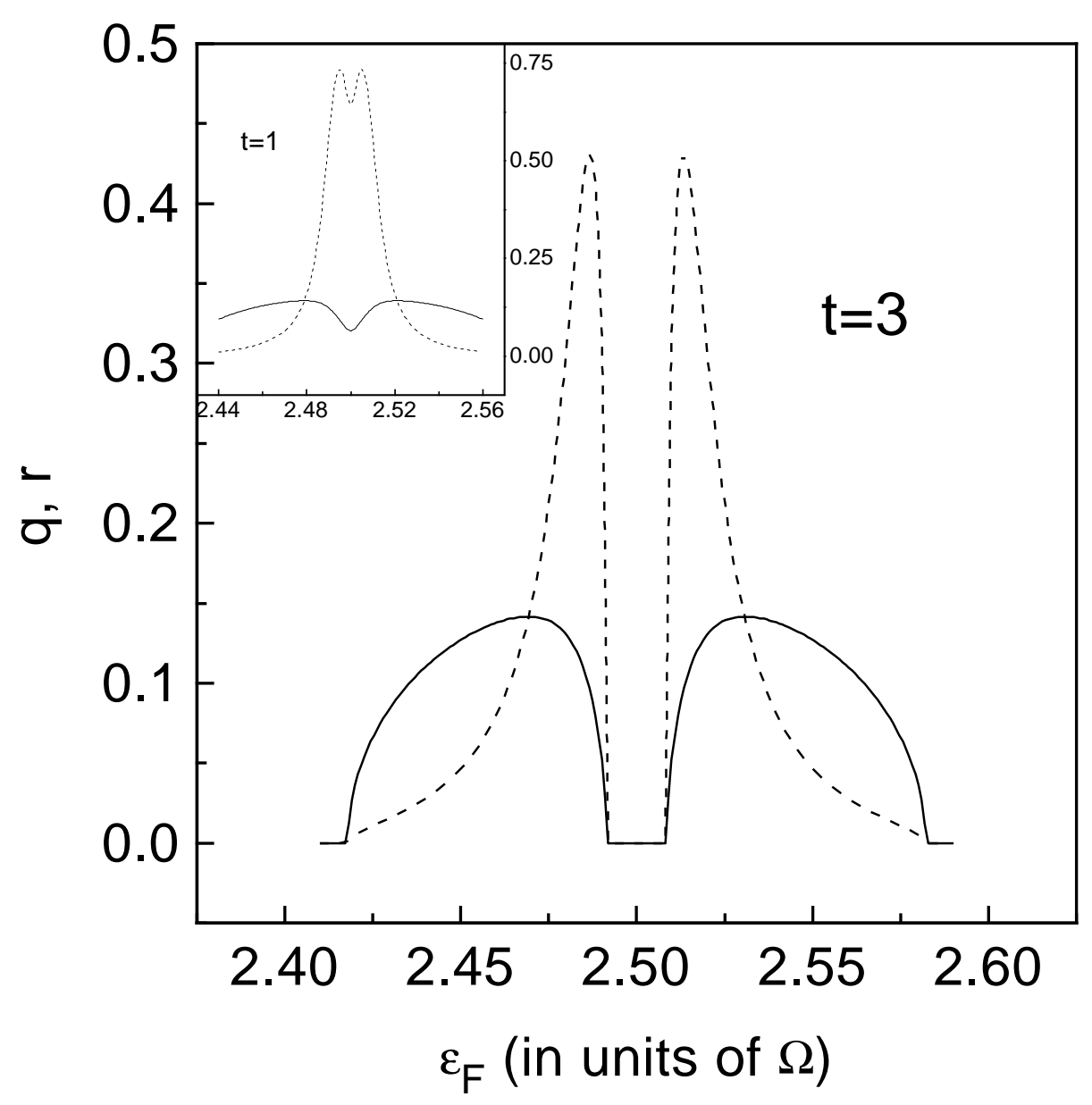

Figure 3 